\section{$\underset{\text { hommes migrations }}{\text { homments }}$}

\section{Hommes \& migrations}

Revue française de référence sur les dynamiques

migratoires

\section{$1322 \mid 2018$}

Exposer les migrations

\title{
Migrations au musée de Bretagne : un regard sociologique
}

\section{Anne Morillon}

\section{OpenEdition}

\section{Journals}

Édition électronique

URL : https://journals.openedition.org/hommesmigrations/6669

DOI : 10.4000/hommesmigrations.6669

ISSN : 2262-3353

\section{Éditeur}

Musée national de l'histoire de l'immigration

\section{Édition imprimée}

Date de publication : 1 juillet 2018

Pagination : 91-99

ISBN : 978-2-919040-42-1

ISSN : $1142-852 X$

\section{Référence électronique}

Anne Morillon, "Migrations au musée de Bretagne : un regard sociologique », Hommes \& migrations [En ligne], 1322 | 2018, mis en ligne le 01 juillet 2020, consulté le 21 janvier 2022. URL : http://

journals.openedition.org/hommesmigrations/6669; DOI : https://doi.org/10.4000/

hommesmigrations.6669 


\section{MIGRATIONS \\ AU MUSÉE DE BRETAGNE : UN REGARD SOCIOLOGIQUE}

Par ANNE MORILLON, docteure en sociologie, Collectif Topik ${ }^{1}$, Rennes.

Présentée en 2013 au Musée de Bretagne, I'exposition Migrations est le fruit d'une collaboration inédite entre une institution patrimoniale et un collectif de sociologues sur le thème des immigrations étrangères en Bretagne. En donnant à voir les liens entre I'histoire de ce territoire abordé sous l'angle de l'émigration et la diversité des origines migratoires de sa population actuelle, cette exposition visait à élargir l'audience du musée. Pour remplir cet objectif, la réflexion commune des professionnels du musée, des chercheurs et des acteurs associatifs locaux a conduit à la création d'un nouveau procédé de médiation à destination des publics du champ social.

La présente contribution est d'abord le témoignage d'une sociologue fortement engagée dans la préparation d'une exposition portant sur l'immigration et l'émigration en Bretagne. Elle constitue également une première analyse des enjeux de l'exposition pour le Musée de Bretagne qui l'a réalisée. Ces derniers sont de deux ordres. Ils portent sur la façon dont une exposition sur la migration, sujet de société s'il en est, a conduit le musée à davantage travailler avec de multiples partenaires et à toucher de nouveaux publics. L'exposition Migrations, présentée entre mars et août 2013, oscille entre mise en scène des souvenirs et entreprise de reconnaissance de la place des émigrés bretons et des immigrés étrangers dans l'histoire passée et présente de la Bretagne. L'exposition montre, à travers des photos, des vidéos, mais aussi de nombreux objets, que la Bretagne est une terre d'émigrations et d'immigrations. Plus d'un million de personnes ont quitté la Bretagne au XIX siècle et lors d'une partie du XX è siècle. En 2013, près de 85000 étrangers, qu'ils soient ouvriers, étudiants ou artistes vivent en région Bretagne et en Loire-Atlantique. L'expérience migratoire est au cœur de l'exposition qui en reprend les grandes « étapes » en s'efforçant de montrer la complexité du phénomène et ses enjeux pour les personnes. En contrepoint, l'attitude de la société dite « d'accueil » est questionnée. Quel accueil ? Quel contrôle? Quel regard porté sur l'autre, l'étranger?

L'exposition met notamment en évidence, avec des titres de transport et autres cartes de séjour, que le contrôle des populations immigrées ici et ailleurs est une préoccupation constante de l'administration policière à partir du début du XX siècle. L'objet de l'exposition est aussi de mettre à l'épreuve, à 
travers diverses situations, l'idée, relayée depuis une dizaine d'années par une partie des médias bretons et sans doute aussi partagée par une partie de l'équipe du musée, d'une "Bretagne, terre d'accueil». Est-ce vrai? Plus ou moins vrai qu'ailleurs ? Il n'est pas si simple de répondre à ces questions. Quoi qu'il en soit, l'exposition Migrations est une invitation à la réflexion sur la Bretagne contemporaine et celles et ceux, dans toute leur pluralité, qui l'ont faite et la font au quotidien.
Enfin, écrire à partir d'une exposition l'histoire d'un territoire nécessite de le définir, ce qui se révèle toujours problématique pour le XIX et le début du $\mathrm{XX}^{\mathrm{e}}$ siècle. Le choix a été fait de prendre en compte le département de la Loire-Atlantique (LoireInférieure jusqu'en 1957) dans le traitement du sujet, sans lui donner autant d'importance qu'aux autres départements de l'actuelle région Bretagne surtout pour les périodes les plus récentes. La Loire-Inférieure, auparavant en Bretagne, a été

\section{Une brève histoire de l'immigration en Bretagne}

Du XIXe siècle à nos jours, les étrangers ont toujours été présents en Bretagne pour travailler ou se réfugier, pour s'installer et « faire souche », malgré leur faiblesse numérique. Territoire économiquement éprouvé, donc faiblement touché par l'immigration (1869 étrangers en 1851 , soit $0,08 \%$ de la population de la région), la Bretagne est toutefois marquée par la présence d'étrangers dans les villes où ils exercent des "spécialités professionnelles » : chocolatiers espagnols, tailleurs et ébénistes allemands, plâtriers figuristes italiens, pâtissiers suisses... C'est aussi une terre de prédilection pour les artistes étrangers, fascinés par la Bretagne " authentique » et " exotique » (Portugais, Scandinaves, Polonais, Russes, Canadiens, Américains...) pour des séjours "touristiques", mais aussi plus durables quand ce sont des réfugiés (Russes, Polonais...). La présence de réfugiés y est, en effet, ancienne (Jacobites irlandais, Acadiens, puis, après les échecs des révolutions nationales en Europe, Piémontais, Italiens, Polonais, Allemands).

Jusque dans les années 1920, les immigrés sont toutefois peu nombreux en Bretagne. Ce décalage chronologique par rapport à d'autres régions françaises est lié aux mutations et aux structures de l'économie régionale, mais aussi aux mouvements de populations internes à la région et à l'exode rural. L'arrivée des travailleurs migrants y est donc d'autant plus faible et plus tardive parce que les besoins de main-d'œuvre ont été dans un premier temps satisfaits par les populations locales. En Bretagne, la Première Guerre mondiale marque une première immigration importante de travailleurs étrangers et de "coloniaux " liée à l'économie de guerre. Pendant l'entre-deux-guerres, les travailleurs étrangers sont présents dans les villes, plus industrialisées, pour combler le déficit de maind'œuvre lié à l'exode rural et à l'émigration bretonne, mais aussi à l'absence de formation technique des «locaux ». L'immigration progresse ainsi dans les années 1920 : entre 1921 et 1926, elle passe de 4196 à 7196 personnes, avec une augmentation remarquable dans le département de l'Ille-et-Vilaine. En 1926, les
Britanniques et les Italiens tiennent le haut du pavé devant les Belges et les Polonais. Les exploitants des carrières de granit ou de grès font par exemple appel à des Italiens. Cette pratique se poursuit dans les années 1930 malgré les mesures affirmant la préférence pour la main-d'œuvre française. De 1926 à 1946, le nombre d'étrangers est stable - environ 7000 personnes - mais les nationalités les plus représentées changent: moins de Britanniques, plus d'Espagnols et d'Italiens. Après le coup d'État du Général Franco, les Espagnols sont nombreux à franchir la frontière. Entre 1936 et 1939, ils sont estimés à 12000 dans les départements de l'Ille-etVilaine, du Morbihan, des Côtes-du-Nord et du Finistère. En 1975, la population étrangère est presque deux fois plus nombreuse qu'en 1968. Sous l'effet conjugué des contextes politiques et économiques des pays de provenance, l'immigration évolue : Espagnols, Portugais, Algériens et Marocains devancent désormais les Italiens. La construction du nouveau quartier sud de Rennes, par exemple, explique en grande partie la progression de l'effectif des Portugais et des Marocains à la fin des années 1960 dans la ville. Dans les années 1970-1980, plus d'un millier de Cambodgiens, Laotiens et Vietnamiens, qui fuient les exactions des dictatures communistes, trouvent refuge en Bretagne, en particulier au foyer Guy Houist à Rennes.

À la fin du XX siècle, le visage de l'immigration en Bretagne se transforme encore, avec une diversification des aires de provenance: Mongolie, Tchétchénie, Europe centrale, Corne de l'Afrique, etc. Plus jeunes que l'ensemble de la population de la région, ces migrants viennent en grande majorité pour demander l'asile ou travailler. L'immigration féminine est également en forte augmentation, tout comme celle des étudiants. Des retours commencent à se dessiner pour les Britanniques qui sont encore les plus nombreux dans la région. Si l'évolution de l'immigration doit être liée pour une large part à l'avenir de l'économie bretonne, le phénomène est d'ores et déjà inscrit dans l'histoire récente de la région. 
rattachée à la "région d’Angers » en 1941 par le gouvernement de Vichy. Cette question éminemment polémique en Bretagne - et au-delà de ses frontières administratives actuelles - est évoquée négativement dans le livre d'or de l'exposition: "Une maladresse dans la présentation la Bzh et la Loire-Atlantique. Ce département n'est-il pas dans la Bzh historique?... »².

\section{Construire l'exposition à partir d'une étude scientifique}

L'exposition est le fruit d'un long travail préparatoire impliquant de nombreux partenaires : personnes migrantes liées ou non à des associations, chercheurs, professionnels d'institutions patrimoniales et culturelles, élus et artistes. Elle s'inscrit aussi dans le prolongement de l'étude Histoire et mémoire de l'immigration en Bretagne réalisée entre 2006 et 2007 à la suite d'un appel à projet national pour des études régionales sur ce sujet, lancé par l'Agence pour la cohésion sociale et l'égalité des chances (Acsé) et la Cité nationale de l'histoire de l'immigration (CNHI), actuel Musée national de l'histoire de l'immigration. Invité à participer au comité de pilotage de l'étude, le Musée de Bretagne s'était montré très intéressé, notamment parce que leur projet d'exposition voulait interroger le territoire breton du point de vue des mouvements de populations (émigrations bretonnes/immigrations étrangères en Bretagne).

La restitution de l'étude en novembre 2008 avait d'ailleurs donné lieu à une journée régionale ${ }^{3}$ au Musée de Bretagne, lors de laquelle il s'agissait déjà de faire dialoguer les deux thèmes des immigrations étrangères et des émigrations bretonnes. Soulignons que, lors de l'étude, la directrice régionale de l'Agence nationale pour la cohésion sociale et l'égalité des chances (Acsé), également ancienne directrice du Fonds d'action et de soutien pour l'intégration et la lutte contre les discriminations (Fasild) en Bretagne, fortement engagée dans la valorisation des histoires individuelles et collectives de l'immigration et la prévention/lutte contre les discriminations, avait souhaité que le comité de pilotage soit le plus ouvert possible, notamment aux institutions patrimoniales et aux équipements culturels, dans la perspective de valoriser l'étude sous diverses formes, dont l'exposition.

\section{De l'immigration aux mouvements de populations}

Avec 104621 immigrés en 2014 (un peu plus de $3 \%$ de la population), la Bretagne fait partie des régions françaises qui disposent du plus faible pourcentage d'immigrés ${ }^{4}$. Mais, entre 1975 et 2014, sa population immigrée a plus que quintuplé, pour devenir ces dernières années un sujet de préoccupation politique et citoyen. Cependant, la pertinence d'un tel thème au cœur d'une exposition au sein d'un musée d'histoire et de société en Bretagne ne s'impose pas d'elle-même. L'argument du nombre d'étrangers, hier comme aujourd'hui, est tantôt invoqué par les médias et une partie des élus du territoire pour minimiser l'importance politique de cette question, tantôt pour la grossir et alimenter les fantasmes d'une «invasion ». L'évolution du phénomène migratoire en Bretagne depuis les années 1970 - qui renvoie aussi à une attractivité générale de la région tant sur un plan économique que culturel et social - est davantage perçue comme un problème que comme un atout. Certains élus préfèrent ainsi l'invisibiliser ou l'ériger en cause des difficultés du territoire. Pour autant, les élus et financeurs du Musée de Bretagne (Rennes 
"La valise d'Hyppolyte ", exposition Migrations, 2013. Fils d'une famille de métayers du Morbihan, il travaillait sur les chantiers parisiens de l'après-guerre avant de tenter l'aventure à l'étranger. Sa valise est un condensé de sa vie d'émigré. CC-BY-SA - Musée de Bretagne, cliché Alain Amet.

métropole, Région Bretagne et DRAC essentiellement) n'ont pas - loin s'en faut - mis leur véto à ce projet.

On peut sans doute faire l'hypothèse que la mise en exposition des immigrations étrangères en Bretagne n'a pu être possible que dans la mesure où elle engageait un dialogue avec le phénomène inverse, si l'on peut dire, de l'émigration des Bretons. Perçue comme un sujet plus légitime dans l'histoire de la Bretagne depuis le XIX siècle, elle a probablement contribué à "légitimer » le sujet de l'immigration étrangère. Dans une certaine mesure, concevoir une exposition aussi ambitieuse sur un sujet aussi sensible que les migrations dans un endroit aussi prestigieux que le Musée de Bretagne n'a pu se faire que parce que le phénomène, à léchelle de la région, était abordé dans son ensemble. Pour autant, ce dialogue "imposé » n'a pas empêché une grande liberté de ton dans le contenu, notamment l'expression à peine voilée d'une critique de la politique d'immigration menée en 2013 par la France et que l'on observe aussi évidemment en Bretagne, faisant dire à certains mécontents que l'exposition avait un parti pris «immigrationiste ${ }^{5}$ ». Ainsi, pour le Musée de Bretagne, faire le choix d'un tel propos, c'était poser « la question des enjeux capitaux d'évolution et de compréhension de la sociétét ». 


\section{Une collaboration entre l'institution patrimoniale et le collectif de sociologues}

La proposition que le collectif Topik a faite en 2010 au directeur du Musée de Bretagne de l'époque a reçu un bon accueil de la part de son équipe. Notre intention première était de donner à voir les résultats de l'étude Histoire et mémoire de l'immigration en Bretagne, tout en l'enrichissant d'une collecte auprès des personnes migrantes vivant en Bretagne aujourd'hui. Pour le musée, cette sollicitation faisait écho au projet déjà ancien d'une exposition consacrée aux émigrations bretonnes. Le propos de l'exposition s'inscrit dans la volonté de porter un regard différent sur le territoire de la Bretagne non plus en termes d'identité(s) comme c'est régulièrement le cas, mais en termes de mouvements de populations. L'exposition alimente ainsi l'idée d'une "Bretagne ouverte sur le monde " qui semble occuper une place croissante dans les représentations que les Bretons ont d'eux-mêmes et de leur région.

Cette approche ouvre des perspectives finalement assez peu travaillées jusque-là : en quoi l'histoire récente de la Bretagne est-elle traversée par ces mouvements de populations? Dans quelle mesure l'expérience des Bretons qui quittent leur terre natale pour une autre région ou un autre pays est-elle semblable à celle vécue par les étrangers qui arrivent en Bretagne? Quels points communs? Quelles différences? Autant de questions qui ont guidé le travail préparatoire et nos échanges avec l'équipe du Musée de Bretagne, en particulier avec Françoise Berretrot, commissaire principale de l'exposition, qui a été notre interlocutrice privilégiée. L'expérience était nouvelle pour nous, sociologues, mais aussi pour le musée, qui avait davantage l'habitude de recruter des "chargés de mission " pour la durée de préparation d'une exposition. Pour ce projet, Angélina Étiemble et moi-même étions liées au musée par une convention en tant que sociologues membres du collectif Topik. Nous avons progressivement trouvé un mode de fonctionnement qui s'est avéré enrichissant pour les deux parties, chacune faisant un pas de côté pour prendre en compte les enjeux, les contraintes et le cadre de travail de l'autre. La mise en œuvre de l'exposition s'est étalée sur trois ans avec une accélération la dernière année lors de laquelle l'une des deux sociologues - moi-même - a été particulièrement associée à toutes les phases du travail, à raison de deux jours par semaine.

\section{Nouveaux partenariats et modalités de travail}

L'exposition Migrations a donc été inaugurée le 14 mars 2013. Au-delà des deux sociologues du collectif Topik associées à son élaboration, elle a mobilisé, à des degrés divers, la participation de trente-cinq chercheurs. Deux cent cinquante-trois personnes ont apporté leur témoignage en répondant au questionnaire "sur la toile» pour les émigrés bretons, en confiant leur histoire ou leurs objets, en acceptant d'être photographiées et filmées pour les autres. Plus de cinquante associations de toute la Bretagne et du monde entier (émigrés bretons) ont été contactées. 16738 personnes ont visité les $600 \mathrm{~m}^{2}$ de l'exposition, soit une moyenne de quatre-vingt-sept personnes par jour, ce qui représente une bonne fréquentation par rapport à d'autres expositions comparables. Elle a été financée par la Direction régionale des affaires culturelles (Drac), pour le volet collectage notamment, Rennes métropole et la région Bretagne. Dans la perspective de l'itinérance de l'exposition et pour enrichir le corpus et les contacts de personnes migrantes susceptibles d'apporter leur témoignage ou des objets, le Musée de Bretagne et les sociologues du collectif Topik ont animé pendant presque deux années des groupes de travail impliquant des acteurs culturels et patrimoniaux locaux, des élus et des associations intéressés pas le projet. Les réunions ont eu lieu à Saint-Brieuc, Vannes et Brest. L'objectif de cette mise en réseau était de sensibiliser les acteurs des institutions patrimoniales à la richesse encore insuffisamment exploitée de leur fonds sur la thématique, de 
repérer les lieux d'accueil pour l'exposition, les associations et les chercheurs susceptibles d'apporter leur contribution au projet. Elle a également facilité le collectage. Pour des raisons financières, l'itinérance de l'exposition souhaitée par le musée n'est pas allée à son terme. La fin du portage par le musée de cette version « nomade » de l'exposition n'a cependant pas découragé les collectivités locales les plus motivées puisque les villes de Brest (service culturel) et de Saint-Brieuc (musée d'art et d'histoire) ont souhaité adapter l'exposition en reprenant certains contenus (textes, films, enregistrements, reportages photographiques) et en empruntant une partie du mobilier. Il s'agissait pour eux de reprendre les grandes lignes de la version initiale et de développer une approche locale, comme ce fut le cas à Brest par exemple pour la partie consacrée aux réfugiés espagnols dont le contenu a été enrichi par une association culturelle espagnole locale.
Les associations dites

" communautaires",

nombreuses en Bretagne,

à Rennes et à Brest en particulier, ont largement été sollicitées, notamment pour la collecte de témoignages et d'objets, le bouche-à-oreille

et les réseaux personnels des différents professionnels engagés ont fait le reste
Il semble que les professionnels du musée ont pu, à l'occasion des trois années de préparation de l'exposition Migrations, développer une diversification de leurs partenaires par le biais de cette nouvelle thématique. Le monde associatif ne leur était pas inconnu bien sûr, mais il renvoyait à des domaines abordés de façon "plus classique " par un musée de société situé en
Bretagne, comme le monde maritime ou les traditions culinaires ou artistiques par exemple. Les associations dites "communautaires », nombreuses en Bretagne, à Rennes et à Brest en particulier, ont largement été sollicitées, notamment pour la collecte de témoignages et d'objets, le bouche-à-oreille et les réseaux personnels des différents professionnels engagés ont fait le reste. La méconnaissance de ces réseaux associatifs par les acteurs du musée a été compensée par l'expertise des chercheurs du collectif Topik ayant une longue pratique d'enquête de terrain et de connaissance des écrits (articles et littérature grise essentiellement) évoquant ces formes d'organisation communautaire dans la région.

\section{Forte sollicitation et assez faible implication des associations de migrants}

Si de nombreuses associations de migrants ont été consultées et mises à contribution, elles n'ont pas pris une part active à la construction de l'exposition, au synopsis et aux choix scénographiques qui, pour des raisons d'efficacité dans la prise de décision, ont reposé essentiellement sur l'équipe du musée, les sociologues de Topik, le scénographe et la graphiste. La volonté de départ était d'associer davantage les associations et les personnes concernées (les migrants et leurs descendants) par le biais de groupes de travail. Mais, pour des raisons qui tiennent peut-être au format de ces réunions, aux difficultés désormais bien connues de "faire participer les habitants " (rapports de pouvoir, sur-représentation des professionnels et des élus, enjeux de s'autoriser à prendre la parole et à donner son avis, à faire part de ses connaissances, modalités d'animation pas nécessairement «inclusives », etc.), mais aussi aux propres dynamiques des associations, ces temps d'échange sont restés largement des temps professionnels. Les associations qui ont pu et voulu participer à la conception de l'exposition sont celles qui avaient déjà eu des projets mémoriels (livres, expositions) que l'ambition régionale de l'exposition Migrations et sa visibilité ont séduit. D'une manière générale, les "petites associations de quartier ", pourtant bien connues et sollicitées, n'ont pas donné suite en raison - on peut le supposer - du caractère descendant du projet, des critiques ayant été formulées çà et là sur une approche trop académique, universitaire ou patrimoniale.

S'il n'est pas question ici de mettre en cause les professionnels - dont nous sommes - dans nos pratiques et points de vue spécifiques, technique ou académique, et si la volonté d'associer les premiers et premières concernées était au cœur du projet de l'exposition, ce sont les modalités de sa mise en œuvre qu'il convient d'interroger. Elles relèvent sans doute de pratiques professionnelles 
Visite commentée de l'exposition Migrations par Pascal Nignol, 2013. CC-BY-SA - Musée de Bretagne, cliché Alain Amet.

à ré-inventer, mais aussi d'une nécessaire attention aux processus de minorisation dont les migrants sont souvent l'objet dans notre pays, impliquant sans nul doute des manières de travailler particulières qui n'ont été mises en œuvre que partiellement pour l'exposition Migrations. Certes, ces difficultés ne sont pas propres aux projets culturels et patrimoniaux, d'autres professionnels, animateurs, travailleurs sociaux, mais aussi artistes, intervenant dans les quartiers populaires, se trouvent confrontés à la nécessité de "faire avec " et non pas "pour " les habitants. Cela suppose chaque fois de repenser les modalités d'une intervention trop souvent verticale ou descendante.

\section{Une approche individuelle de la migration ?}

La participation des associations de migrants pose aussi la question de la place des mémoires de l'immigration et de la prise en compte des dynamiques collectives dans le parcours de l'exposition à côté, en illustration ou en contrepoint des archives documentaires. La participation pleine et entière des personnes migrantes et de leurs descendants suppose de partager des expériences et des souvenirs directs ou indirects, et de débattre sur la place qu'il convient de leur accorder dans une exposition qui balaie un siècle de présence étrangère et de migration intérieure en ou depuis la 
Bretagne. En l'absence, d'une manière générale, d'associations dites "communautaires", le choix s'est de fait porté sur une approche de l'expression mémorielle davantage individuelle que collective, fondée sur des témoignages sensibles recueillis oralement ou filmés, des photographies, des objets venant illustrer un récit socio-historique issu de l'étude Histoire et mémoire de l'immigration en Bretagne et de nombreuses monographies consacrées à telles ou telles populations de l'immigration, telle période ou tel territoire. Ce choix vise également à provoquer chez le visiteur de l'empathie et à fournir une respiration dans l'appréhension d'un propos parfois âpre et scientifique. L'idée de s'attacher à des destins individuels est particulièrement visible dans la mise en valeur de l'histoire d'Hyppolyte à partir de sa valise. Fils d'une famille de métayers du Morbihan, il travaillait sur les chantiers parisiens de l'après-guerre avant de tenter l'aventure à l'étranger. Sa valise est un condensé de sa vie d'émigré. S'y trouvent,

Assurées par l'équipe

du musée, ces formations

ont porté, d'une part,

sur le musée et l'exposition

Migrations et, d'autre part, sur le dispositif les « relais du champ social » et ses enjeux pour les professionnels $\mathrm{du}$ secteur social et patrimonial. pêle-mêle, des dizaines de souvenirs de Bretagne et d'ailleurs : carte de bibliothèque de San Francisco, plan de Montréal, broderie des chutes du Niagara... Par ailleurs, on retrouve cette fonction empathique dans le projet Une boîte, une histoire, réalisée par des élèves de $5^{\mathrm{e}}$ du collège Les Quatre moulins de Brest, à partir de la collecte d'objets et de photographies de leurs parents et aïeuls. Dans cet univers restreint, ils reconstituent leurs histoires familiales avec leurs mots, leur subjectivité, leur poésie. Présentées ensemble, ces boîtes forment un kaléidoscope de paysages et d'origines : des quatre coins de Bretagne en passant par Mayotte, le Cameroun, l'Égypte... En s'attachant à ces personnes, le visiteur est ainsi amené à entrer dans un sujet difficile et éminemment politique. Finalement, on retrouve ici de manière très concrète le paradoxe de l'invisibilité/visibilité de l'histoire de l'immigration, qui tend à privilégier les trajectoires singulières au risque de masquer les processus sociologiques de hiérarchisation ethno-raciale, de stigmatisation et de relégation sociale et spatiale.

\section{Amener de nouveaux publics au musée}

À l'occasion de l'exposition Migrations, une nouvelle forme de médiation a été mise en place par le Musée de Bretagne : les « relais du champ social ». Ce dispositif, inspiré de la mission "Vivre ensemble $e^{7}$ ", prévoit la construction de partenariats avec les principaux équipements sociaux du département d'Ille-et-Vilaine. Les publics du «champ social» sont, d'une part, les personnes en situation de précarité (économique, isolement social, maladie) et, d'autre part, celles qui ne sont pas considérées comme « intégrées » : étrangers, handicapés, illettrés, détenus... Le dispositif consiste à former des personnes relais (travailleurs sociaux, animateurs, professionnels ou bénévoles...) pour accompagner ces publics, considérant que, pour les amener au musée, la gratuité n'est pas suffisante. Elle doit être assortie d'un accompagnement spécifique de professionnels ou de bénévoles qu'ils côtoient régulièrement et avec lesquels ils ont instauré une relation de confiance. Une médiation adaptée et interactive pendant la visite est, selon les acteurs de cette forme de médiation, essentielle : «Sortir ensemble, passer un bon moment et partager nos émotions est une façon de rompre l'isolement. Créer du lien social permet de développer une autonomie et de se sentir plus enraciné dans la société ou de s'approprier la ville en douceur pour se sentir bien ${ }^{8}$. "Trente-quatre structures sociales, socio-éducatives ou culturelles du département d'Ille-et-Vilaine ont participé à ce dispositif pendant les six mois de présentation de l'exposition. Trois sessions de formation de deux heures ont formé soixante-neuf « relais » et un peu plus de la moitié ont effectivement proposé aux usagers de leurs équipements une visite de l'exposition. 
Assurées par l'équipe du musée, ces formations ont porté, d'une part, sur le musée et l'exposition Migrations et, d'autre part, sur le dispositif les « relais du champ social » et ses enjeux pour les professionnels du secteur social et patrimonial.

\section{Le bilan du dispositif expérimental}

Le bilan qualitatif, établi par une chargée de mission du musée recrutée pour la mise en œuvre de ce dispositif, fait état de constats qui peuvent être élargis à d'autres actions ayant trait au traitement social et politique des « publics précaires ». En effet, du côté des associations "relais » chargées de la mise en œuvre concrète de ces visites avec l'équipe de médiation du musée, l'évaluation positive insiste sur la gratuité instaurée par le dispositif, ainsi que sur tous les autres avantages « en nature » (affiche, catalogue de l'exposition, carte d'abonnement d'un an offerte par le musée). De son côté, l'équipe du musée souligne la plus-value d'un accompagnement social allant au-delà d'une médiation «classique » qui ne prend pas forcément en compte les attentes et les besoins spécifiques de ces publics. Toutefois, ces appréciations positives sont nuancées par les personnes chargées du « relais » qui alertent sur les risques de stigmatisation de l'expression « visiteurs du champ social » figurant explicitement sur la pancarte informative à l'accueil. Elles craignent finalement l'institutionnalisation de ce type de visites organisées et dédiées spécifiquement à ce public précis, au détriment d'une véritable démarche "d'inclusion». Les professionnels du musée soulignent, quant à eux, à la fois la charge de travail supplémentaire que ce dispositif entraîne et la nécessité de créer un poste dédié ; certains évoquent aussi une certaine frilosité de l'équipe, peu habituée à accueillir ce type de visiteurs. Malgré ces réserves, le bilan fait état d'une "fréquentation remarquable » dans ce cadre avec cinq-cent-soixante visiteurs. Ce nouveau procédé de médiation a ensuite suscité de nouvelles attentes, les associations ou les équipements relais exprimant clairement l'envie de renouveler cette expérience lors de prochaines expositions.

L'exposition Migrations fut pour le Musée de Bretagne l'occasion de toucher un plus large public, notamment les habitants des quartiers populaires et les immigrés. La méconnaissance du réseau des associations, notamment communautaires, évoquée précédemment est, depuis l'exposition Migrations, comblée tant par la préparation que par la médiation « relais du champ social », ces associations ayant été parmi les premières à visiter l'exposition.

\section{Sortir de sa «zone de confort »}

La thématique des migrations a eu un effet de levier, incitant les professionnels à se lancer dans la mise en place cette médiation: émigration/ immigration sont des sujets susceptibles d'attirer un autre public, à côté des visiteurs habituels, à la condition de penser l'accueil et l'accompagnement des nouveaux publics, comme le permet le dispositif « relais du champ social ». Cependant, au-delà de l'intérêt d'une telle initiative dont le bilan du musée se fait l'écho, on peut s'interroger sur cette idée d'une « thématique adaptée » à des publics dits " éloignés de la culture », qui laisserait entendre que les sujets plus classiques pour un musée seraient alors inadaptés.

Quoi qu'il en soit, force est de constater qu'en acceptant de consacrer une exposition d'une telle ampleur à un sujet jugé à la fois politiquement sensible et minoritaire en Bretagne et en proposant une médiation peu habituelle, l'équipe du musée n’a pas hésité à prendre des risques. Notre principale interlocutrice, conservatrice de profession, a évoqué plusieurs fois avec nous les réactions d'hostilité vis-à-vis du sujet de quelques membres de l'équipe. Avec l'exposition Migrations, le musée s'est éloigné de sa "zone de confort», mais - et finalement tous en conviennent - il a rempli pleinement son rôle de musée de société questionnant un sujet devenu, même en Bretagne, incontournable. 